Special Issue: Cutting-Edge Research on Intestinal Immunity and Inflammation

Mini Review

\title{
Induction of humoral responses by epidermal Langerhans cells
}

\section{Keisuke Nagao}

Dermatology Branch, Center for Cancer Research, National Cancer Institute, National Institutes of Health, Bethesda, MD, U.S.A.

Langerhans cells (LC) are the outermost immunological sentinels of mammalian organisms and represent the unique dendritic cell subset in epidermis. LC have been the focus of vigorous research, but their physiological roles are just beginning to be elucidated. While LC are clearly potent antigen presenting cells in vitro, demonstration of in vivo functions had been challenging. This short review will summarize a series of some recent work that has uncovered the ability of LC to induce humoral responses after antigen capture via tight junctions, a process that confers systemic immunity against antigens that have not yet breached epidermal barriers. This process, which we refer to as "preemptive" immunity, might also be relevant for percutaneous sensitization in allergic skin diseases.

Rec.9/19/2014, Acc.10/15/2014, pp23-27

Correspondence should be addressed to:

Keisuke Nagao, Dermatology Branch, Center for Cancer Research, National Cancer Institute, National Institutes of Health, Bldg 10 Rm4N222, 9000 Rockville Pike, Bethesda, MD 20892-1908, U.S.A. Phone: +1-301-496-2481, Fax: +1-301-496-5370, E-mail: keisuke.nagao@nih.gov

Key words Langerhans cells, dendritic cells, tight junctions, antigen capture, humoral response, preemptive immunity

\section{Introduction}

After their discovery by Paul Langerhans in 1868, and the discovery of dendritic cells (DC) by Ralph Steinman in $1973^{1)}$, epidermal Langerhans cells (LC) were demonstrated to be bone marrow-derived leukocytes ${ }^{2}$ that express class II $\mathrm{MHC}^{3)}$, and were capable of stimulating $\mathrm{T}$ cells ${ }^{4)}$, therefore belonging to the DC subset. A long list of reports, in which functional studies were performed mostly in vitro, established the LC paradigm ${ }^{5}$, which held that LC captured antigens that were encountered in skin and migrated to skin-draining lymph nodes to activate antigen-specific $\mathrm{T}$ cells.

It therefore came as a surprise when several groups revisited this paradigm in transgenic mouse models in vivo, and in aggregate, failed to demonstrate a requirement for LC in initiating immune responses in two different settings. The first report demonstrated that LC were not required for anti-viral $C D 8^{+} \mathrm{T}$ cell responses to percutaneous herpes virus 
infection ${ }^{6}$, and additional studies showed that LC were not essential for eliciting hapten-induced contact hypersensitivity responses $^{7-9}$. It is now established that $\mathrm{CD} 103^{+} \mathrm{DC}$, which include the langerin ${ }^{+}$dermal $D C$, represent a DC subset that most efficiently cross-presents antigens to $\mathrm{CD}^{+} \mathrm{T}$ cells ${ }^{10)}$, and that LC appear to be minimally involved during such an immune response. Langerin ${ }^{+}$dermal DC share langerin expression with LC, but are peripheral blood-derived and exist in the dermis, and unlike LC, do not depend on TGF- $\beta$ for development.

The involvement of LC in responses to haptens is still somewhat controversial. Constitutive or transient depletion of LC utilizing Langerin-DTA (diphtheria toxin fragment A) or Langerin-DTR (diphtheria toxin receptor) transgenic mice $^{7,8)}$ has resulted in either increased ${ }^{8)}$, decreased ${ }^{11)}$ or unchanged $^{9)}$ contact hypersensitivity responses. These differences may result from the type of hapten used and/ or the timing of DT treatment, which can lead to different kinetics or efficiency of repopulation by langerin+ dermal DC, as will be introduced below.

One limitation with the usage of haptens during studies on LC function is that topically applied haptens readily penetrate through the two epidermal barriers, stratum corneum and tight junctions ${ }^{12}$, then through epidermis into dermis, and can persist locally for 2 or 3 days. Thus, can haptens not only engage epidermal and dermal resident DC subsets, but they can also be acquired by newly recruited blood-derived monocytes and DC that could contribute to the complexity of downstream immune responses. Although contact hypersensitivity is clinically relevant and experiments in mice have taught us much about skin immunity, it may not be the optimal system to explore LCspecific functions.

\section{LC elicit antigen-dependent IgG1 re- sponses against gene gun immunized bacterial antigens in vivo}

Skin is inhabited by vast varieties of microbes, most of which are non-pathogenic, but some species such as Staphylococcus aureus can exert strong pathogenicity and cause serious infections. Because LC are positioned in epidermis close to the outside environment, we hypothesized that LC survey skin and elicit immune responses against potentially pathogenic microbes among the skin microbiota. Because of the complexity of the skin barriers, however, we initially avoided this issue and utilized gene gun to introduce bacterial antigen into skin to determine if
LC were capable of inducing a specific immune response. Gold particles (covered with cDNA encoding antigens of interest) "shot" via gene gun readily penetrates into epidermis and dermis, resulting in transient $(<24 \mathrm{hrs})$ expression of proteins of interest.

After transient depletion of langerin-expressing DC subsets in Langerin-DTR mice with diphtheria toxin (DT), langerin ${ }^{+}$dermal DC repopulate the dermis in $\sim 7$ days, whereas it takes LC at least 4 weeks to repopulate the epidermis. Taking advantage of the different repopulation kinetics, Langerin-DTR mice were treated with DT 13 days (lacking LC only) or 1 day (lacking both LC and langerin ${ }^{+}$ $\mathrm{dDC}$ ) before single-time immunization with $\beta$-galactosidase cDNA via gene gun ${ }^{13}$. Analyzing serum two weeks later by ELISA revealed decreased $\beta$-galactosidase-specific IgG1 responses in mice that lacked LC, and decreased $\operatorname{lgG} 1$ and IgG2c in mice that lacked both LC and langerin ${ }^{+}$ dermal $D C^{13)}$. This result indicated that $L C$ were important for the induction of IgG1 and langerin ${ }^{+}$dermal DC for IgG2C responses. Thus, LC and the newly discovered langerin ${ }^{+}$ dermal DC were distinct DC subsets that were capable of inducing distinct immune responses in vivo ${ }^{13)}$.

\section{Antigen capture through tight junctions}

Two layers of epidermal barriers, the stratum corneum and the tight junctions, inhibit passive penetration of antigens $^{12}$. Stratum corneum is an insoluble structure that consists of three functionally distinct layers, and provides the outermost physical barrier on skin surface ${ }^{14)}$. Tight junctions function as selective barriers between aqueous compartments to maintain osmotic balance and transport other solute substances, but they do not allow paracellular passage of macromolecules such as protein antigens ${ }^{15}$. Although localization of skin microbes on or within the superficial layers of skin is not completely understood, it is likely that they exist outside of tight junctions.

If LC were to actively survey and gain access to skin microbes or microbial antigens present on intact skin, they would be required to penetrate their dendrites through the tight junction barrier. Indeed, dendritic cells in the gut ${ }^{16)}$ and the airway ${ }^{17}{ }^{18)}$ extend their dendrites through simple epithelia to capture antigens from the lumen. We therefore asked if LC were capable of capturing foreign material that had breached the stratum corneum, but not the epidermal tight junction barrier.

Gentle tape stripping (which removes several layers of stratum corneum) induced the activation of LC, all of which 
extended their dendrites upward and appeared to dock with, or penetrate, the tight junction barrier ${ }^{19)}$. Topical application of a membrane-impermeable biotinylation reagent enabled visualization of endocytosis that occurred at dendrite tips of LC that had penetrated the tight junction barrier. Furthermore, LC were capable of capturing ovalbumin as well as $E$. coli that was applied via occlusive dressings onto tape stripped mouse ears ${ }^{19}$. During this process of tight junction penetration, LC appeared to express tight junction proteins Claudin-1 and ZO-1, suggesting that tight junction barriers are formed between LC and keratinocytes to maintain barrier integrity during surveillance.

Thus, this study demonstrated that LC were capable of capturing potential antigens via dendrite penetration through tight junctions ${ }^{19)}$, but whether LC subsequently induced specific immune responses was yet to be established.

\section{Langerhans cell induction of preemptive immunity}

To extend the above two studies and to demonstrate LC function in a more biological setting, an experimental model of staphylococcal scalded skin syndrome (SSSS) was established. SSSS is a potentially fatal childhood disease that is caused by exfoliative toxin (ET)-producing $S$. aureus infection. ET reaches distant skin sites via the blood stream, and cleaves desmoglein 1 (Dsg1), a major component of desmosomes on keratinocytes in superficial epidermis, that leads to the loss of keratinocyte-keratinocyte adhesion, thereby causing severe blistering ${ }^{20)}$. When recombinant ETA ( $r E T A$ ) was administered intraperitoneally into wildtype mice, it rapidly created erythematous lesions that were Nikolsky's sign-positive (detachment of skin upon gentle rubbing) with histological features identical to that of human SSSS, thereby recapitulating the human disease ${ }^{21)}$.

As expected for a protein antigen, ETA did not penetrate tight junction barriers. When ETA was injected intradermally, despite the expression of Dsg1 below and above tight junctions, blisters were always formed immediately below tight junctions ${ }^{21}$. Furthermore, ETA did not create local lesions when applied via occlusive dressing onto tape stripped mouse ears (a procedure we will refer to as patch-immunization in this manuscript). Staining such patchimmunized lesions for tight junction proteins revealed normal expression, collectively supporting the conclusion that ETA does not penetrate through tight junction barriers ${ }^{21)}$. Thus, immune responses occurring after patch-immunization should be a result of antigen capture via tight junction by

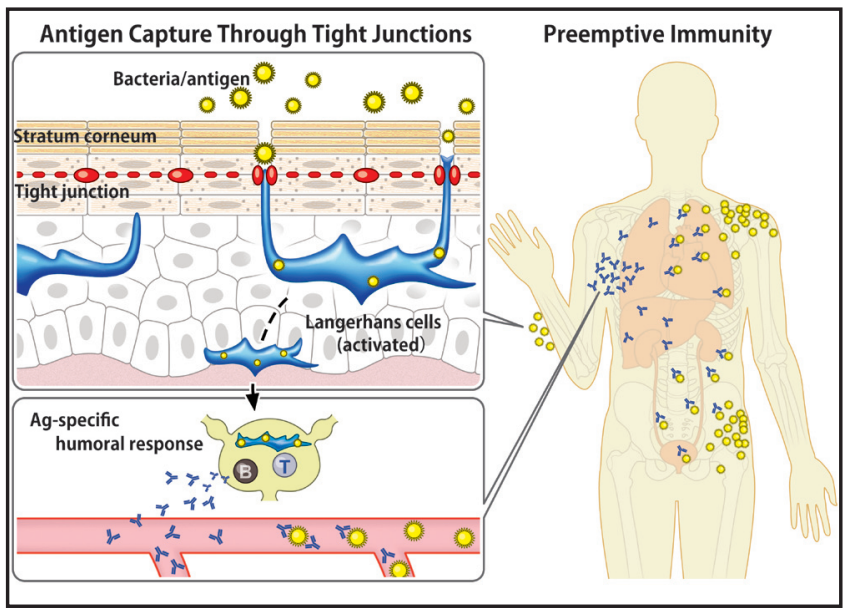

Fig. 1 Langerhans cells confer preemptive immunity In response to stimuli, activated LC elongate their dendrites through epidermal tight junctions to acquire antigens that have breached the stratum corneum. LC then induce antigen-specific neutralizing antibodies that confer systemic immunity against captured antigens ${ }^{27)}$.

LC.

Indeed, when rETA was given i.p., ETA-specific IgG1, 2 , and 4 were induced ${ }^{21)}$. In contrast, patch-immunization with rETA lead to the selective production of ETA-specific $\lg \mathrm{G} 1$, and this response was eradicated when LC were depleted prior to patch-immunization in Langerin-DTR mice ${ }^{21)}$. These results indicated that LC were capable of capturing antigens via tight junctions and preferentially induced lgG1 responses (Fig. 1). rETA patch-immunized mice, but not unimmunized mice, were protected from developing SSSS when given rETA injection ${ }^{21)}$. Importantly, Langerin-DTR mice that were depleted of LC prior to patch-immunization with ETA developed SSSS, demonstrating an important role for LC in conferring host defense against antigens that were captured through tight junctions ${ }^{21)}$. It was confirmed that LCinduced ETA-specific IgG1 had neutralizing capabilities in vitro $^{21)}$.

\section{Discussion and Conclusion}

The three studies summarized herein collectively show that LC survey skin for protein antigens that exist outside of tight junctions by extending their dendrites through this barrier, and then induce neutralizing antibodies predominantly of the IgG1 subclass. These findings are consistent with the existence of anti-ETA antibodies in humans who have never contracted SSSS ${ }^{22)}$, and it is possible that the biological process we have identified in mice also takes place in humans. IgG1 was the only prominent response 
that was detected in the patch-immunization setting utilizing both OVA and ETA ${ }^{21)}$. However topical application of OVA with dibutyl phthalate ${ }^{23)}$ or OVA conjugated with fluorescein isothiocyanate ${ }^{24)}$ has induced OVA-specific $\lg E$ responses. Although it is not clear whether OVA breaches the tight junction barrier in such a setting, it is conceivable that perturbation of epidermis elicits signals that directly or indirectly act on LC to induce antibody responses accompanied by class or subclasses other than IgG1.

Antigen capture via tight junctions by LC has mechanistic implications regarding protein antigen sensitization through skin in allergic diseases. Recent outbreak of wheatdependent exercise-induced anaphylaxis in Japan caused by hydrolyzed wheat-containing soap $^{25)}$ emphasizes the importance of percutaneous sensitization that is likely mediated by LC. Chronic perturbation in the epidermis of atopic dermatitis patients might also elicit signals that enhance humoral responses to antigens that are acquired by LC through barrier-disrupted skin.

How or where LC capture antigens might influence the subsequent immune responses that they elicit. When microbes such as Candida invade through skin barriers and breach the epidermis, LC induce $\mathrm{TH} 17$ responses ${ }^{26)}$. Utilization of protein antigens has elucidated LC functions that are distinct from those of other skin DC subsets, and these functions might also be relevant to humans in clinical settings. The involvement of LC in tolerance is being actively explored, but results have been inconclusive. It is possible that LC capture not only foreign antigens, but also keratinocyte-associated self-antigens. It would be of interest to pursue regulatory functions of LC utilizing self (protein)antigens to elucidate possible roles for LC in autoimmunity.

\section{Sources of funding}

This research was supported by the Intramural Research Program of the $\mathrm{NIH}$.

\section{Conflict of interests}

No conflicts of interest to be disclosed.

\section{Reference}

1) Steinman RM, Cohn ZA: Identification of a novel cell type in peripheral lymphoid organs of mice: I. Morphology, quantitation, tissue distribution. J Exp Med. 1973; 137: 1142-1162.

2) Katz SI, Tamaki K, Sachs DH: Epidermal Langerhans cells are derived from cells originating in bone marrow.
Nature. 1979; 282: 324-326.

3) Klareskog L, Tjernlund UM, Forsum U, Peterson PA: Epidermal Langerhans cells express la antigens. Nature. 1977; 268: 248-250.

4) Schuler G, Steinman RM: Murine epidermal Langerhans cells mature into potent immunostimulatory dendritic cells in vitro. J Exp Med. 1985; 161: 526-546.

5) Wilson NS, Villadangos JA: Lymphoid organ dendritic cells: beyond the Langerhans cells paradigm. Immunol Cell Biol. 2004; 82: 91-98.

6) Allan RS, Smith CM, Belz GT, van Lint AL, Wakim LM, Heath WR, Carbone FR: Epidermal viral immunity induced by CD8alpha+ dendritic cells but not by Langerhans cells. Science. 2003; 301: 1925-1928.

7) Bennett CL, Noordegraaf M, Martina CA, Clausen BE: Langerhans cells are required for efficient presentation of topically applied hapten to T cells. J Immunol. 2007; 179: 6830-6835.

8) Kaplan DH, Jenison MC, Saeland S, Shlomchik WD, Shlomchik MJ: Epidermal Langerhans cell-deficient mice develop enhanced contact hypersensitivity. Immunity. 2005; 23: 611-620.

9) Kissenpfennig A, Henri S, Dubois B, Laplace-Builhé C, Perrin P, Romani N, Tripp $\mathrm{CH}$, Douillard P, Leserman L, Kaiserlian D, Saeland S, Davoust J, Malissen B: Dynamics and function of Langerhans cells In vivo: dermal dendritic cells colonize lymph node areas distinct from slower migrating Langerhans cells. Immunity. 2005; 22: 643-654.

10) Bedoui S, Whitney PG, Waithman J, Eidsmo L, Wakim L, Caminschi I, Allan RS, Wojtasiak M, Shortman K, Carbone FR, Brooks AG, Heath WR: Cross-presentation of viral and self antigens by skinderived CD103+ dendritic cells. Nat Immunol. 2009; 10: 488-495.

11) Bennett CL, van Rijn E, Jung $S$, Inaba K, Steinman $\mathrm{RM}$, Kapsenberg ML, Clausen BE: Inducible ablation of mouse Langerhans cells diminishes but fails to abrogate contact hypersensitivity. J Cell Biol. 2005; 169: 569-576.

12) Kubo A, Nagao K, Amagai M: Epidermal barrier dysfunction and cutaneous sensitization in atopic diseases. J Clin Invest. 2012; 122: 440-447.

13) Nagao K, Ginhoux F, Leitner WW, Motegi S, Bennett CL, Clausen BE, Merad M, Udey MC: Murine epidermal Langerhans cells and langerin-expressing dermal dendritic cells are unrelated and exhibit 
distinct functions. Proc Natl Acad Sci USA. 2009; 106: 3312-3317.

14) Kubo A, Ishizaki I, Kubo A, Kawasaki H, Nagao K, Ohashi $Y$, Amagai $\mathrm{M}$ : The stratum corneum comprises three layers with distinct metal-ion barrier properties. Sci Rep. 2013; 3: 1731.

15) Günzel D, YAS L: Claudins and the Modulation of Tight Junction Permeability. Physiological Reviews. 2013; 93: 525-569.

16) Rescigno $M$, Urbano $M$, Valzasina $B$, Francolini $M$, Rotta G, Bonasio R, Granucci F, Kraehenbuhl J-P, RicciardiCastagnoli P: Dendritic cells express tight junction proteins and penetrate gut epithelial monolayers to sample bacteria. Nat Immunol. 2001; 2: 361-367.

17) Jahnsen FL, Strickland DH, Thomas JA, Tobagus IT, Napoli S, Zosky GR, Turner DJ, Sly PD, Stumbles PA, Holt PG: Accelerated Antigen Sampling and Transport by Airway Mucosal Dendritic Cells following Inhalation of a Bacterial Stimulus. J Immunol. 2006; 177: 5861-5867.

18) Thornton EE, Looney MR, Bose O, Sen D, Sheppard D, Locksley R, Huang X, Krummel MF: Spatiotemporally separated antigen uptake by alveolar dendritic cells and airway presentation to $\mathrm{T}$ cells in the lung. $\mathrm{J}$ Exp Med. 2012; 209: 1183-1199.

19) Kubo A, Nagao K, Yokouchi M, Sasaki H, Amagai M: External antigen uptake by Langerhans cells with reorganization of epidermal tight junction barriers. J Exp Med. 2009; 206: 2937-2946.

20) Amagai M, Matsuyoshi N, Wang ZH, Andl C, Stanley JR: Toxin in bullous impetigo and staphylococcal scaldedskin syndrome targets desmoglein 1. Nat Med. 2000; 6: 1275-1277.

21) Ouchi T, Kubo A, Yokouchi M, Adachi T, Kobayashi T, Kitashima DY, Fujii H, Clausen BE, Koyasu S, Amagai M, Nagao K: Langerhans cell antigen capture through tight junctions confers preemptive immunity in experimental staphylococcal scalded skin syndrome. J Exp Med. 2011; 208: 2607-2613.

22) Nishioka K, Nakano T, Hirao N, Teranishi H, Asada Y: Staphylococcal scalded skin syndrome. II. Serum level of anti exfoliatin and anti alpha-toxin in patients with staphylococcal scalded skin syndrome or bullous impetigo. J Dermatol. 1977; 4: 65-68.

23) Kawasaki H, Nagao K, Kubo A, Hata T, Shimizu A, Mizuno $H$, Yamada T, Amagai M: Altered stratum corneum barrier and enhanced percutaneous immune responses in filaggrin-null mice. J Allergy Clin Immunol. 2012; 129: 1538-1546.e1536.

24) Nakajima S, Igyártó BZ, Honda T, Egawa G, Otsuka A, Hara-Chikuma M, Watanabe N, Ziegler SF, Tomura M, Inaba K, Miyachi Y, Kaplan DH, Kabashima K: Langerhans cells are critical in epicutaneous sensitization with protein antigen via thymic stromal lymphopoietin receptor signaling. J Allergy Clin Immunol. 2012; 129: 1048-1055.e1046.

25) Chinuki Y, Takahashi H, Dekio I, Kaneko S, Tokuda R, Nagao M, Fujisawa T, Morita E: Higher allergenicity of high molecular weight hydrolysed wheat protein in cosmetics for percutaneous sensitization. Contact Dermatitis. 2013; 68: 86-93.

26) Igyártó BZ, Haley K, Ortner D, Bobr A, Gerami-Nejad M, Edelson BT, Zurawski SM, Malissen B, Zurawski G, Berman J, Kaplan DH: Skin-resident murine dendritic cell subsets promote distinct and opposing antigenspecific T helper cell responses. Immunity. 2011; 35: 260-272.

27) Nagao K: Hair follicles and Langerhans cells. Enshou To Men'eki (Inflammation \& Immunity). 2014; 22: 171-175. 